\title{
FUZZY LOGIC IN REAL ESTATE VALUATION
}

\author{
Victoria López ${ }^{1}$, Álvaro del Monte ${ }^{1}$, Javier Montero ${ }^{2}$ \\ ${ }^{1}$ Fac. Informatics, Complutense University, Madrid, Spain. \\ 2 Fac. Mathematics, Complutense University, Madrid, Spain.
}

\begin{abstract}
Real estate valuation is a complex problem where experts make their job subject to an input information that is mostly fuzzy in nature. In this paper we present a real estate valuation model that allows the management of such a fuzzy information. In particular, we realize that our reference set of experts considers at a first stage a division of all potential clients into four main clusters, according to their preferences. An inference engine is then designed in order to obtain the degree as valuation of a real state described by the client and their own characteristics. A relevant feature of our proposal is that we allow fuzzy specifications.
\end{abstract}

\section{Introduction.}

Buyers and renters make their decisions according to a set of preferences, based upon an analysis that pursues in some way to determine which real estate within a certain fits those preferences. First of all, a general expert elaborates a real estate form for describing all relevant characteristics of each real estate (size, rooms, views, etc.) and a basic classification of all potential clients (buyers and renters). Such a real estate form is compounded by all criteria and characteristics being relevant for client's decisions. In our case, data will be stored into a data base (Microsoft Access or SQL), which will be ready to accept queries from the real estate valuation tool.

Classification of potential clients implies a cluster match with a description of the ideal real estate for such a type of client. According to our general expert, we should be considering five basic clusters: investors; 2-3 children family; single male; single female; young couple. Since investment is very sensible to a lot of factors, in this paper we limit the analysis to the other four clusters. Descriptions of the clusters are done by means of fuzzy first order logic, as specification of requirements in any system. Evaluations of specifications are done by means of a functional tool for fuzzy first order logic evaluation [1], called XFuzzy [5]. XFuzzy will allow an automatic 
generation of code in different languages of programming (Java, $\mathrm{C}++, \mathrm{C}$ ), to be used in any inference. An additional program independent program is developed for management and valuations.

The paper is organized as follows: in section 2 we remind some basic fuzzy concepts, in particular the XFuzzy 3.0 tool; in section 3 we describe the exact terms of the real estate valuation problem we face; in section 4 we present the design of our model, including the associated decision making criteria and its inference rules. Last section 5 is devoted to some conclusions and future work.

\section{Fuzzy specification.}

As pointed out in [3], projects in software engineering need in general a lot of requirement specification, to be made by means of documents, tables and data-flow models, among others. The practical relevance requirement specification processes is a standard within classical software development. This argument also applies to a fuzzy context, see [4]. The introduction of fuzzy techniques is a must whenever software process deals with some kind of fuzzy uncertainty. This was the main argument in [2], where a real estate valuation was developed by means of a particular funcional tool for fuzzy first order logic (see [5]). Instead of this tool, in this paper we consider XFuzzy 3.0 tool, created by the National Center for Microtechnoly of Spain, [5]. XFuzzy is an environment for developing fuzzy logic inference systems. Two main characteristics of XFuzzy are its great flexibility and capacity for developing complex systems.

The fuzzy system developed in this paper use the following XFuzzy stages: (1) Xfuzzy design stage (definition of inference base rules and membership functions); (2) Verification stage (a monitoring tool should take care of a proper working system); and (3) Synthesis stage (an automatic generation of code of the fuzzy system being designed). In this paper we have chosen a Java implementation in order to link with the valuation program developed in Java as well.

\section{Real state valuation problem.}

Since our main goal is the estimation of the valuation of a real estate, we can not apply classical approaches, at least meanwhile this kind of problem is highly dependent on the perception value that the buyer or the renter have about the real estate under consideration. But first of all it is necessary to obtain all data of interest, determine the preferences of the customer, 
and develop a fuzzy system being able to manage valuation of real estate with respect to such preferences.

As any other decision making problem, not all characteristics or properties about a real estate are relevant in a first approach. In this paper we shall consider a set of 20 basic characteristics, that we first divide into two, depending on their crisp or fuzzy nature. The subset of crisp characteristics contains numerical description like the number of rooms, size, garage, and so on. Fuzzy characteristics are qualitative and no easily associated to an integer number (nice views, good light, comfortability, and so on. Our general expert defines not only both subsets of characteristics but also the form in which all this data will be taken. For example, about lift, it is enough to take 'yes' if there exists or 'no' otherwise. However, a fuzzy characteristic like 'good light' must be valuated by an expert (someone who has a good knowledge about the flat or apartment). The value assigned to a fuzzy characteristic had to be chosen between 0 (the worst) and 10 (the best). Later on a fuzzy set is also used to define this type of characteristics. Of course these data can be given by a unique expert, but in this particular work data where given by two different experts (characteristics structure was given by the general estimations, but a second expert assigned estimated values).

Moreover, despite each buyer or renter has a personal preference, our general expert establishes four different classes of customers that define differentiate preferences in their search for a flat or apartment: (1) $\varphi_{1}$ : young couple; (2) $\varphi_{2}$ : 2-3 children family; (3) $\varphi_{3}$ : single male; and (4) $\varphi_{4}$ : single female. All experts we consulted basically agree with this classification, at least for a first approach, in the sense that all their present clients fitted one of these groups. Hence, in this paper we decided to refuse non-typical clients, just in case someone not fitting those four classes could appear. In addition, a set of primary characteristics (fuzzy or crisp) will be associated to each client profile:

- $\varphi_{1}: 2$ bedrooms, downtown, garage and middle price

- $\varphi_{2}$ : at least 3 bedrooms, garage, garden and pools, good light, in the suburbs area.

- $\varphi_{3}$ : small apartment (1 bedroom), garage, downtown, middle price.

- $\varphi_{4}: 2$ bedrooms, good light, elevator, garage if possible, below middle price.

Finally, it must be pointed out that sorting preferences is in general a difficult issue, even if the number of items is not too low, but it becomes critical when dealing with fuzzy criteria. In this paper we have considered 
diagrams of preferences for determining the degree being assigned to each characteristic. These diagrams are developed by an expert according to the environment and each client profile, reminding Hasse diagrams as a preorder relation between criteria. Each client profile is characterized by a diagram by means of an easy and intuitive information model close to natural language without loosing too much information.

\section{The fuzzy system design.}

This section deals with the design of a fuzzy system that returns the valuation of a real estate according to the client preferences. In our model, the system uses as input data nine primary parameters that come up out from the union of all primary characteristics in all profiles: type of estate (flat, apartment, villa, etc.), area, number of rooms, good light, lift, garage, housing development, community features and cost. A variable defined into the fuzzy system is associated to each parameter. The system generates then four output variables according to the valuation of the real estate with respect to each profile. In a first stage XFuzzy requires a fuzzyfication of the input variables by defining membership functions. Such a fuzzyfication allows a faster computation in a fuzzy framework.

A membership function is then related to an input variable in order to point out the degree to which characteristics carry out certain property. Next figures show some examples of membership functions: figure 1 shows the range of variables taking values between 0 and 10 (for good light or community features, for example, we may choose between labels like 'no important', 'little important', 'not little important', 'important', 'very important', in order to represent the degree of fulfillment of each client about each variable; figure 2 has only three labels, one per each profile (except the 2-3 children profile, which does not show the characteristic cost as a primary characteristics; and last figure 3 shows the membership function for the four output variables. This time it is defined by means of five lables: 'very bad', 'bad', 'regular', 'good' and 'very good'. Hence, the set of fuzzy inference rules will be defined taking into account preferences for each profile in the following sense: let $P=\left\{C_{1}, \ldots, C_{k}\right\}$ be the set of criteria that fits with the profile of the client. If an apartment satisfy all criteria then this apartment must be valuated as a 'very good' for this profile. If the apartment doesn't satisfy any one, then it will be 'very bad' valuated. If it satisfies a few criteria, then it will be valuated as 'bad', and so on (all these rules are made by the designer in XFuzzy 3.0). 


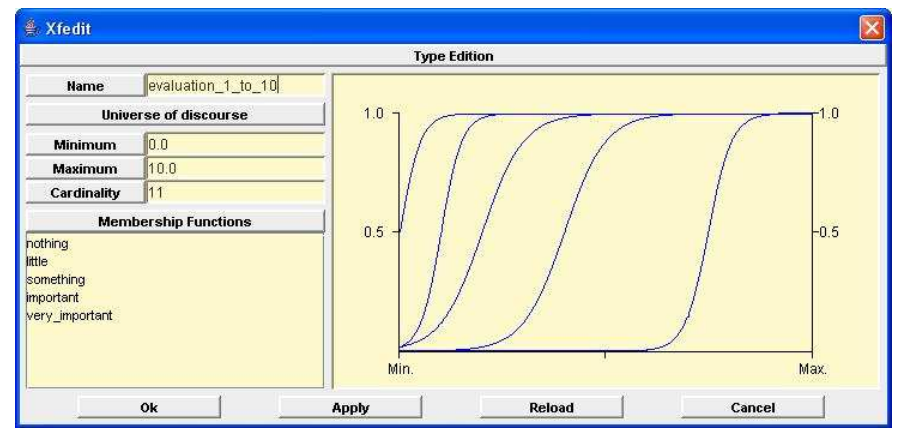

Fig. 1. XFuzzy 3.0 design flow.

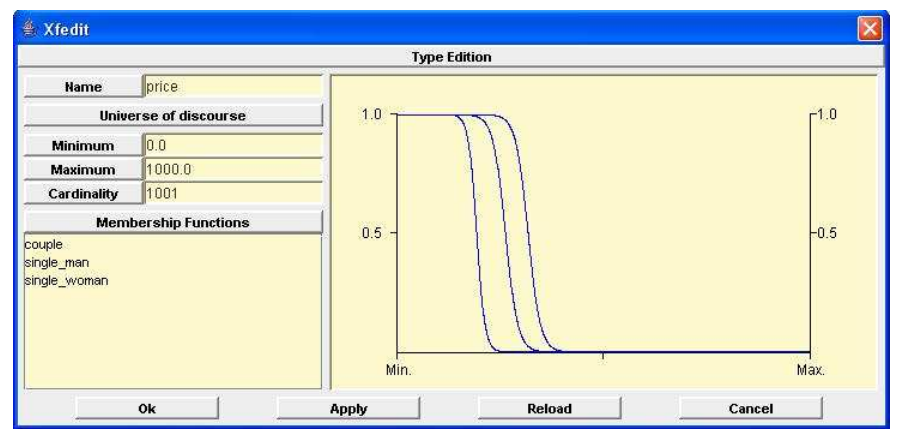

Fig. 2. XFuzzy 3.0 design flow.

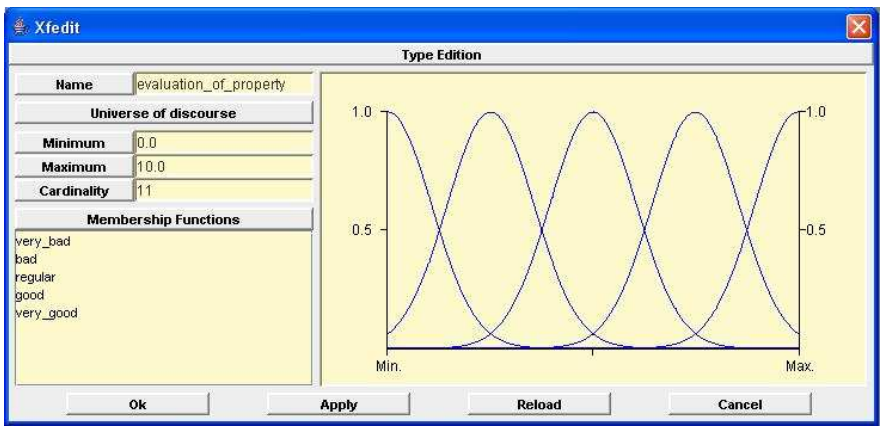

Fig. 3. XFuzzy 3.0 design flow. 


\section{Program.}

All data, functions and inferences above introduced have allow us to develop a specific software where valuations are assigned according to an specific profile of client. Such a software has been developed in Java (XFuzzy is able to create or generate automatically the code, which was then added to the software implementation), making not only valuations, but also adding, editing and deleting data about estates (flats, apartments, villas, ...) By means of this software, users can make queries about valuations and search the item that better fits with a given profile.

\section{Conclusions and future work.}

Many decision making problems need fuzzy logic, since fuzziness is present in quite a number of key concepts that appear when human beings manage information. This paper shows a particular potential application, still to be developed, when real estate has to be valuated according to the profile of potential buyers or renters. In order to get such a valuation, we have distinguished several phases (definition of data and variables, membership functions and fuzzyfication, definition of appropriate fuzzy inference rules into the model, and computing the value of the output variables (defuzzification). Although we are in a very preliminary stage, a software tool with the above outlined characteristics will be hopefully very useful for any estate business.

Acknowledgments: This Research has been partially supported by grant TIN2006-06190, Government of Spain.

\section{References}

1. V. López, J. M. Cleva and J. Montero, A functional tool for Fuzzy First Order Logic Evaluation. In D. Ruan et al., eds. Applied Artificial Intelligence (World Scientific, New Jersey, 2006); pp. 19-26.

2. V. López and J. Montero, Fuzzy specification of algorithms, Proceedings of the New Trends in Preference Modeling, Eurofuse Workshop (A Demanda, Jaén, 2007); pp. 151-156.

3. V. López, J. Montero, Fuzzy specification in software engineering, Proceedings of the Joint Conference on Infomation Sciences (World Scientific, Salt Lake City, 2007).

4. J. Montero, V. López and D. Gómez, The role of fuzziness in decision making. In D. Ruan et al., eds.: Fuzzy Logic: an spectrum of applied and theoretical issues (Springer, Berlin, 2007).

5. XFuzzy 3.0. Centro Nacional de Microelectrnica. Instituto de Microelectrnica de Sevilla. Http://www.imse.cnm.es/xfuzzy 